\title{
The Impact of Focused Attention on Emotional Experience: A Functional MRI Investigation
}

\author{
Florin Dolcos ${ }^{1,2,3} \cdot$ Yuta Katsumi ${ }^{1,2} \cdot$ Chen Shen $^{1,2} \cdot$ Paul C. Bogdan ${ }^{1,2} \cdot$ Suhnyoung Jun ${ }^{1,2} \cdot$ Ryan Larsen ${ }^{1}$. \\ Wendy Heller ${ }^{1,2,3} \cdot$ Kelly Freeman Bost ${ }^{4} \cdot$ Sanda Dolcos ${ }^{1,2}$
}

Published online: 7 August 2020

(C) The Psychonomic Society, Inc. 2020

\begin{abstract}
Emotional well-being depends on the ability to adaptively cope with various emotional challenges. Most studies have investigated the neural mechanisms of emotion regulation strategies deployed relatively later in the timing of processing that leads to full emotional experiences. However, less is known about strategies that are engaged in earlier stages of emotion processing, such as those involving attentional deployment. We investigated the neural mechanisms associated with self-guided Focused Attention $(F A)$ in mitigating subjective negative emotional experiences. Functional magnetic resonance imaging (fMRI) data were recorded while participants viewed a series of composite negative and neutral images with distinguishable foreground (FG) and background (BG) areas. Participants were instructed to focus either on the FG or BG components of the images, and then rated their emotional experiences. Behavioral results showed that FA was successful in decreasing emotional ratings for negative images viewed in BG Focus condition. At the neural level, the BG Focus was associated with increased activity in regions typically implicated in top-down executive control (dorsolateral prefrontal cortex and lateral parietal cortex) and decreased activity in regions linked to affective processing (amygdala and ventrolateral prefrontal cortex). Dissociable brain activity linked to FA also was identified in visual cortices, including between the parahippocampal and fusiform gyri, showing increased versus decreased activity, respectively, during the BG Focus. These findings complement the evidence from prior FA studies with recollected emotional memories as internal stimuli and further demonstrate the effectiveness of self-guided FA in mitigating negative emotional experiences associated with processing of external unpleasant stimuli.
\end{abstract}

Keywords Affect $\cdot$ Emotion control $\cdot$ Amygdala $\cdot$ Prefrontal cortex $\cdot$ Emotion-cognition interactions

Florin Dolcos and Yuta Katsumi Shared First-Authorship

Florin Dolcos

fdolcos@illinois.edu; http://dolcoslab.beckman.illinois.edu

Sanda Dolcos

sdolcos@illinois.edu; http://dolcoslab.beckman.illinois.edu

1 Beckman Institute for Advanced Science \& Technology, University of Illinois at Urbana-Champaign, 405 North Mathews Avenue, Urbana, IL 61801, USA

2 Department of Psychology, University of Illinois at Urbana-Champaign, Champaign, IL, USA

3 Neuroscience Program, University of Illinois at Urbana-Champaign, Urbana, IL, USA

4 Family Resiliency Center, Department of Human Development and Family Studies, University of Illinois at Urbana-Champaign, Urbana, IL, USA

\section{Introduction}

Research on emotion regulation (ER) - the processes influencing which, when, and how emotions are experienced and expressed - has established that the ability to cope adaptively with emotionally challenging situations is vital for both physical and mental health (Gross, 2008, 2015). The process model of ER, proposed by Gross (1998), distinguishes four families of ER strategies based on the emotion generation stage they are primarily affecting. Situational strategies, which seek to change emotion generation at the situation stage, by selecting which situations are encountered or by modifying the situation; Attentional Deployment strategies, which seek to change emotion generation by changing the aspects to which individuals attend to in a situation; Cognitive strategies, which involve changing emotion generation at the appraisal stage, by modifying the way individuals look at/construe the situation; finally, Response Modulation strategies, which seek to change emotion at the 
response stage, by modifying aspects of the interoceptive or exteroceptive world through overt or covert behavior.

Although significant progress has been made in elucidating the neurobehavioral mechanisms of ER over the past decade (e.g., reviewed in Dolcos et al., 2017; Ochsner, Silvers, \& Buhle, 2012; Sheppes, Suri, \& Gross, 2015), prior neuroimaging studies have most commonly focused on examining cognitive strategies, especially cognitive reappraisal, which involves attempts to change the meaning of stimuli or situations, and a response modulation strategy, such as expressive suppression, which involves attempts to decrease emotionally expressive behavior ${ }^{1}$ (Gross, 2008). Recent investigations have begun to reveal the efficacy of the attentional deployment family of ER strategies, typically involving shifts in attention away from emotional aspects of the stimulus, or away from the stimulus altogether, in order to alter emotional responses (Gross, 2008; Sheppes et al., 2014). In particular, one type of attentional deployment strategies, called focused attention (FA), has been proven quite effective in reducing the negative impact of emotional memory encoding (Dolcos et al., 2020b) and retrieval (Denkova, Dolcos, \& Dolcos, 2015; Iordan, Dolcos, \& Dolcos, 2019). Despite its promise as an effective ER strategy, however, it remains unclear how engaging FA influences the immediate subjective experience of emotions, and how the engagement of this particular ER strategy is linked to changes in activity in regions typically associated with emotion processing and regulation. We investigated the neural mechanisms associated with the impact of self-guided FA on subjective emotional experience using functional magnetic resonance imaging (fMRI) in a sample of healthy adults. Clarifying the neurobehavioral mechanisms of self-guided FA can improve our understanding of alterations in affective disorders, which are typically characterized by excessive attention to negative emotional stimuli and debilitating rumination on negative memories.

\section{Attentional Deployment as an Emotion Regulation Strategy}

Available evidence confirms the efficacy of attentional deployment strategies while also identifying their unique advantages over different types of ER strategies. Recent studies have demonstrated the effectiveness of specific types of attentional deployment strategies in regulating emotions (Wadlinger \& Isaacowitz, 2011; Webb, Miles, \& Sheeran, 2012), both in promoting adaptive emotional responses to

\footnotetext{
${ }^{1}$ Departing from Gross's definition, some studies used "suppression" or "suppress" to refer to cognitive reappraisal, either in instructions given to participants or in their description of results (e.g., Phan et al., 2005; Urry, 2009).
}

negative stimuli presented in laboratory settings (Ferri, Schmidt, Hajcak, \& Canli, 2013; Urry, 2010) and in improving the efficacy of longer-term fear reduction interventions (Johnstone \& Page, 2004; Oliver \& Page, 2003, 2008). Several fMRI studies also have begun to reveal the neural mechanisms associated with the impact of engaging such strategies (Dolcos et al., 2020b; Dorfel et al., 2014; Ferri et al., 2013; Kanske, Heissler, Schonfelder, Bongers, \& Wessa, 2011; McRae et al., 2010; for recent meta-analysis, see Morawetz et al., 2017). Notably, attentional deployment strategies such as FA may be considered more efficient than other strategies such as cognitive reappraisal in controlling emotional responses, because their fast deployment enables intervention during earlier stages of the emotion-generative sequence (Paul, Simon, Kniesche, Kathmann, \& Endrass, 2013; Thiruchselvam, Blechert, Sheppes, Rydstrom, \& Gross, 2011). In fact, current evidence suggests that attentional deployment is effective in modulating emotional responses even when it is engaged substantially after the onset of emotion-eliciting stimuli (Sheppes \& Meiran, 2007). This makes attentional deployment particularly useful in real-life situations in which individuals may unexpectedly encounter highly emotional stimuli. In addition, attentional deployment may also be a useful tool for those who have limited cognitive resources. When given a choice to use different ER strategies in downregulating emotional responses to negative stimuli, those participants exhibiting lower executive control abilities were more likely to choose an attentional deployment strategy over reappraisal (Scheibe, Sheppes, \& Staudinger, 2015).

Among the ER strategies typically classified as being part of the attentional deployment family, FA may be especially well suited for prompt regulation of emotional responses in the natural environment. As an attention-based ER strategy, previous studies have demonstrated the efficacy of cognitive distraction, which involves the engagement of cognitive tasks to distract from and mitigate the impact of negative emotions (Kanske et al., 2011; McRae et al., 2010; NolenHoeksema, Wisco, \& Lyubomirsky, 2008; Sheppes, Scheibe, Suri, \& Gross, 2011). For instance, in the face of an anxiety-inducing stimulus (e.g., roller-coaster ride), one might choose to close his eyes and think about the time they felt happy and remember all the details associated with the event, to distract themselves from emotions triggered by the imminent rush of adrenaline. However, it is important to note that using cognitive distraction as an ER strategy typically requires access to a sufficiently demanding secondary task, which may not be readily available in everyday life. FA, involving attentional shifts between emotional and nonemotional aspects within the experienced context, may therefore be a more practical means to regulate emotions in a variety of real-world situations. 


\section{Self-guided Focused Attention as an Emotion Regulation Strategy}

Current evidence regarding the impact of FA on subjective emotional experience and its associated neural mechanisms is still scarce (Ferri et al., 2013; Urry, 2010). The few studies examining the neurobehavioral mechanisms of FA instructed participants to process emotional scenes by artificially introducing effects that do not exist in real life, thus reducing the ecological validity of the manipulations. For instance, in one study participants were asked to view emotional images under an arousing or a non-arousing focus, the latter of which was achieved by prompting them to look at and pay attention to non-arousing areas marked by a circle superimposed on each image (Ferri et al., 2013). In another study, participants were prompted to look at the targeted (e.g., nonemotional) component of each image within which all other (e.g., emotional) areas were blurred (Urry, 2010). Both of these manipulations resulted in reliable changes in subjective emotional experience by directing participants to look at certain highlighted aspects of the stimulus after its onset (i.e., externally-guided FA), but again these approaches have limited applicability beyond the laboratory setting. Altogether, converging evidence suggests that attentional deployment strategies, including cognitive distraction and externally-guided FA, may be highly useful, but a more ecologically valid manipulation of FA may be to instruct participants to guide their attention to focus on different aspects of an emotion-eliciting stimulus in a self-guided manner (Dolcos et al., 2020a).

To our knowledge, no published study has investigated the neural mechanisms associated with the effect of self-guided FA on subjective emotional experience associated with the viewing of emotion-eliciting stimuli. However, the aforementioned investigations of externally-guided FA (Ferri et al., 2013; Urry, 2010) and of cognitive distraction (Kanske et al., 2011; McRae et al., 2010), along with previous studies from our group examining self-guided FA in autobiographical recollection (Denkova, Dolcos, \& Dolcos, 2013a, 2013b; Denkova et al., 2015; Iordan et al., 2019), all demonstrated that the engagement of these attention-based ER strategies is associated with modulation of activity in brain regions typically involved in top-down control and bottom-up emotion processing. Specifically, externally guided focus on nonarousing aspects of emotional images (Ferri et al., 2013) was associated with increased activity in regions including the dorsolateral prefrontal cortex (dlPFC) and the lateral parietal cortex (LPC), typically implicated in top-down executive control, coupled with decreased activity in regions including the amygdala (AMY) and visual association areas, typically activated during basic emotion processing. In addition, the engagement of self-guided FA during autobiographical recollection showed that instructing participants to focus away from emotional aspects and on other nonemotional contextual aspects (e.g., when/where the event happened, who else was involved) was also associated with increased activity in regions associated with ER and regions involved in processing contextual details (parahippocampal place area [PPA]), coupled with decreased activity in the AMY (Denkova et al., 2015; Iordan et al., 2019).

Overall, while differences can be noted, these findings are consistent with available evidence concerning the neural correlates of other ER strategies, such as cognitive reappraisal and emotional suppression (Dorfel et al., 2014; Kohn et al., 2014), suggesting that various ER strategies are in part subserved by a common network of brain regions, with topdown control regions likely modulating activity in bottom-up emotional and perceptual regions. Notably, these opposing patterns of neural activity in regions associated with topdown vs. bottom-up processing are overall consistent with the involvement of large-scale neural systems associated with executive control (e.g., dIPFC, LPC, as part of a dorsal executive system) vs. emotion processing (e.g., AMY, as part of a ventral affective system), linked to the processing of and coping with emotional distraction (Anticevic, Repovs, \& Barch, 2010; Dolcos et al., 2006; Dolcos \& McCarthy, 2006; for reviews, see Dolcos, Iordan, \& Dolcos, 2011; Iordan, Dolcos, \& Dolcos, 2013). That is, reduced distraction is associated with incresed activity in executive regions and reduced activity in basic emotion processing regions. It should be noted that the dorso-ventral dissociation linked to top-down vs. bottom-up processing is not absolute, as some of the ventral $\mathrm{PFC}$ regions, both lateral (ventrolateral $\mathrm{PFC}, \mathrm{vlPFC}^{2}$ ) and medial (ventromedial PFC, vmPFC), also are involved in top-down processing of external (Iordan et al., 2013) and internal emotional stimuli (Denkova et al., 2015; Iordan et al., 2019).

\section{The Present Study}

Despite the potential advantages of self-guided FA over other ER strategies, it remains unclear how engaging this strategy influences the subjective experience of emotions associated

\footnotetext{
It is important to note that, likely due to the anatomical and functional heterogeneity associated with this region (Cai, Ryali, Chen, Li, \& Menon, 2014; Gordon et al., 2016; Levy \& Wagner, 2011; Power et al., 2011; Warren et al., 2014), the vlPFC has been implicated in both generation/ upregulation and appraisal of emotion (Lindquist, Wager, Kober, BlissMoreau, \& Barrett, 2012; Morawetz et al., 2017; Phan, Wager, Taylor, \& Liberzon, 2002; Wager et al., 2008) and executive control/downregulation of emotion (e.g., Badre \& Wagner, 2007; Buhle et al., 2014; Iordan, Dolcos, \& Dolcos, 2013; Kober et al., 2008; Kohn et al., 2014; Morawetz et al., 2017; Ochsner et al., 2012; Wager, Davidson, Hughes, Lindquist, \& Ochsner, 2008). Also, consistent the vlPFC's heterogeneity, evidence from our investigations of emotional distraction points to subregional specificity of these areas (generally overlapping with the inferior frontal cortex), linked to various aspects of affective processing (e.g., basic vs. regulation) (Dolcos, Kragel, Wang, \& McCarthy, 2006; Dolcos \& McCarthy, 2006; reviewed in Iordan et al., 2013).
} 
with processing of external emotional stimuli, and how the engagement of this particular ER strategy is linked to changes in brain activity in regions typically associated with emotion processing and regulation. Clarification of this issue is important in further understanding the utility of self-guided FA as an ER strategy and the associated neural mechanisms with increased specificity. To fill this important gap in the literature, the present study investigated whether the subjective emotional experience associated with viewing external emotional stimuli (i.e., images) is mitigated by engaging self-guided FA. The associated neural mechanisms were investigated by recording $\mathrm{AMRI}$ data during an emotional rating task, during which participants were shown composite negative and neutral images with distinguishable foreground (FG) and background $(\mathrm{BG})$ areas and were instructed to focus either on the FG or BG content of the images.

Based on the available evidence reviewed above, we formulated the following hypotheses regarding the impact of self-guided FA on the subjective experience of emotions and the associated neural mechanisms. First, we expected that focusing on the contextual aspects and away from the emotional aspects of negative images would reduce the subjective emotional experience associated with the viewing of these images. Second, at the neural level, we expected that focusing on the contextual aspects of negative images would be associated with increased activity in fronto-parietal regions (dlPFC, lateral parietal cortex [LPC]), implicated in top-down attentional/executive control, and decreased activity in the AMY, implicated in bottom-up emotion processing. Third, aside from this dissociation between emotion control and emotion processing regions, we also tested the hypothesis that focusing on the contextual aspects of negative images would be associated with differential activity in category-selective higher-order visual processing areas, such as the PPA, which has been identified in studies of self-guided FA with internal stimuli (Denkova et al., 2015; Iordan et al., 2019).

\section{Methods}

\section{Participants}

A total of 24 adult females participated in this study $\left(M_{\text {age }}=\right.$ $\left.34.0, S D_{\text {age }}=4.76\right)$. Only females were recruited because they were part of a larger study investigating mother-child dyads. All participants were healthy, right-handed, native English speakers ( $88 \%$ white, $8 \%$ hispanic, $4 \%$ black), with no recent history of psychiatric or neurological conditions. Data from one participant were excluded from the behavioral analysis due to software malfunction during data collection, and data from three additional participants also were excluded from the eye-tracking, behavioral, and fMRI analyses due to outlier responses in their emotional ratings (based on a $z$ - standardization criterion of $>2.5$ ) (Seo, 2006). Hence, the behavioral analyses were performed on data from 20 participants $\left(M_{\text {age }}=33.85, S D_{\text {age }}=4.82\right)$, and the eye-tracking and fMRI analyses were performed on data from 21 participants $\left(M_{\text {age }}=\right.$ 34.24, $\left.S D_{\text {age }}=4.90\right)$. All participants provided written informed consent under a protocol approved by the Institutional Review Board at the University of Illinois. Each participant was financially compensated for the time spent in the study ( $\$ 15 /$ hour for time spent in the MRI scanner and $\$ 10 /$ hour for time spent outside of the scanner), over two sessions lasting approximately 2 hours each. During the first session, participants performed an emotional rating task in the MRI scanner, and the second session involved behavioral assessments, which also included a surprise memory test (see below).

\section{Emotional Rating Task with FA Manipulation}

Functional MRI and eye-movement data were recorded while participants viewed and rated a total of 90 composite images (60 negative and 30 neutral). Each composite image was created by overlaying a negative or neutral FG component upon a visually complex BG component. The FG components were extracted from images part of the International Affective Picture System (IAPS; Lang, Bradley, \& Cuthbert, 2008), the Geneva Affective Picture Database (GAPED; DanGlauser \& Scherer, 2011), the Military Affective Picture System (MAPS; Goodman, Katz, \& Dretsch, 2016), the Nencki Affective Picture System (NAPS; Marchewka, Zurawski, Jednorog, \& Grabowska, 2014), and the Emotional Picture Set (EmoPicS; Wessa et al., 2010). Images from these sources, along with domainfree online image databases, were used as BG components. Negative and neutral composite images were matched for human presence, animacy, FG location (i.e., top, bottom, left, right), complexity, brightness, and contrast (all $p$ values $>0.05$ ). To obtain data regarding the basic affective properties of these images, we conducted a validation study using an independent sample of participants $\left(N=16 ; 10\right.$ females; $M_{\text {age }}=$ $19.30, S D_{\text {age }}=1.30$ ), who were asked to passively view and rate these images in terms of perceived valence, arousal, and visual complexity on 9-point Likert scales. Based on the data obtained from this validation sample, we made sure that our negative and neutral images were significantly different both in terms of valence and arousal. Specifically, negative images were reliably more negatively valenced $\left(M_{\text {Valence }}=2.46, S D_{\text {Valence }}=\right.$ $0.79)$ and more arousing $\left(M_{\text {Arousal }}=4.95, S D_{\text {Arousal }}=\right.$ $1.05)$ than the neutral images $\left(M_{\text {Valence }}=4.79, S D_{\text {Valence }}\right.$ $\left.=0.48 ; M_{\text {Arousal }}=2.17, S D_{\text {Arousal }}=0.46\right)\left(p_{s}<0.001\right)$. We also made sure that the images assigned to the $\mathrm{FG}$ 
and $\mathrm{BG}$ conditions did not differ in these properties (all $\left.p_{s}>0.05\right)$.

The pool of 90 images was divided into sets of images that were randomly assigned to five study runs, counterbalanced across image type and attentional cue categories. The run orders were randomly assigned to the participants. To avoid negative mood induction, the order of trials was counterbalanced within each run, such that no more than three images of the same emotional category or cue type were presented consecutively. Each image was presented for $4 \mathrm{~s}$ and then was removed to minimize possible confounding effects of eye movements associated with prolonged scanning of images (Figure 1). Approximately 4 days later, participants returned for a surprise memory test. Analyses of the data acquired during this followup session have been reported elsewhere (Dolcos et al., 2020b).

Participants were asked to view each image under different attentional manipulation conditions, cued by the preceding instruction screen. The cues, presented for $0.5 \mathrm{~s}$, directed them to focus either on the image foreground (FG Focus), which was emotional or neutral, or on the image background (BG Focus), which was always neutral. Following the cue, each negative and neutral image was presented for $4 \mathrm{~s}$. Half of both negative and neutral images were preceded by FG Focus cues and the other half by BG Focus cues, and the focus cues were counterbalanced across participants. Following image presentation, participants were asked to rate their subjective emotional experience triggered by the images on a 5 -point scale $(1=N o t$ Negative at All, $5=$ Very Negative). All responses were made on a response pad attached to the participant's right hand. The trial ended with an inter-trial interval of $9.5 \mathrm{~s}$, allowing the hemodynamic response to return to baseline.

\section{Eye-Tracking Data Acquisition}

To assess participants' gaze patterns during the emotional rating task, eye positions and movements were recorded from each participant's right eye using an MR-compatible model (with a long-range mount) of the Eyelink1000 system (SR Research, ON, Canada), at a sampling rate of 1,000 Hz. A pseudorandom 9-point calibration was performed at the beginning of the experimental session and after every other experimental block. The size of the LCD monitor was 23.25 (height) by 41.33 (width) inches, which corresponded to approximately 8.94 (height) and 15.89 (width) degrees of visual angle at the participant's viewing distance (to the mirror) of approximately 3 inches.

\section{fMRI Data Acquisition}

Scanning was conducted on a $3 \mathrm{~T}$ Siemens MAGNETOM scanner with a 64-channel head coil, at the Beckman Institute's Biomedical Imaging Center. After the sagittal localizer and the 3D MPRAGE anatomical images $(\mathrm{TR}=$ $2000 \mathrm{~ms} ; \mathrm{TE}=2.25 \mathrm{~ms} ;$ flip angle $=8^{\circ} ; \mathrm{FOV}=230 \times 230$ $\mathrm{mm}^{2}$, matrix size $=256 \times 256 \mathrm{~mm}^{2} ;$ slice thickness $=1 \mathrm{~mm}$; volume size $=172$ slices; voxel size $=1 \mathrm{~mm}$ isotropic), 5 blocks/runs of whole-brain EPI functional images were acquired axially with a simultaneous multi-slice (SMS) sequence $\left(\mathrm{TR}=1,500 \mathrm{~ms}, \mathrm{TE}=30 \mathrm{~ms}\right.$; flip angle $=40^{\circ} ; \mathrm{FOV}$ $=230 \times 230 \mathrm{~mm}^{2} ;$ matrix size $=144 \times 144 \mathrm{~mm}^{2}$; slice thickness $=1.6 \mathrm{~mm}$; volume size $=76$ slices; multi-band acceleration factor $=4$, voxel size $=1.6 \mathrm{~mm}$ isotropic; phase encoding direction from anterior to posterior).

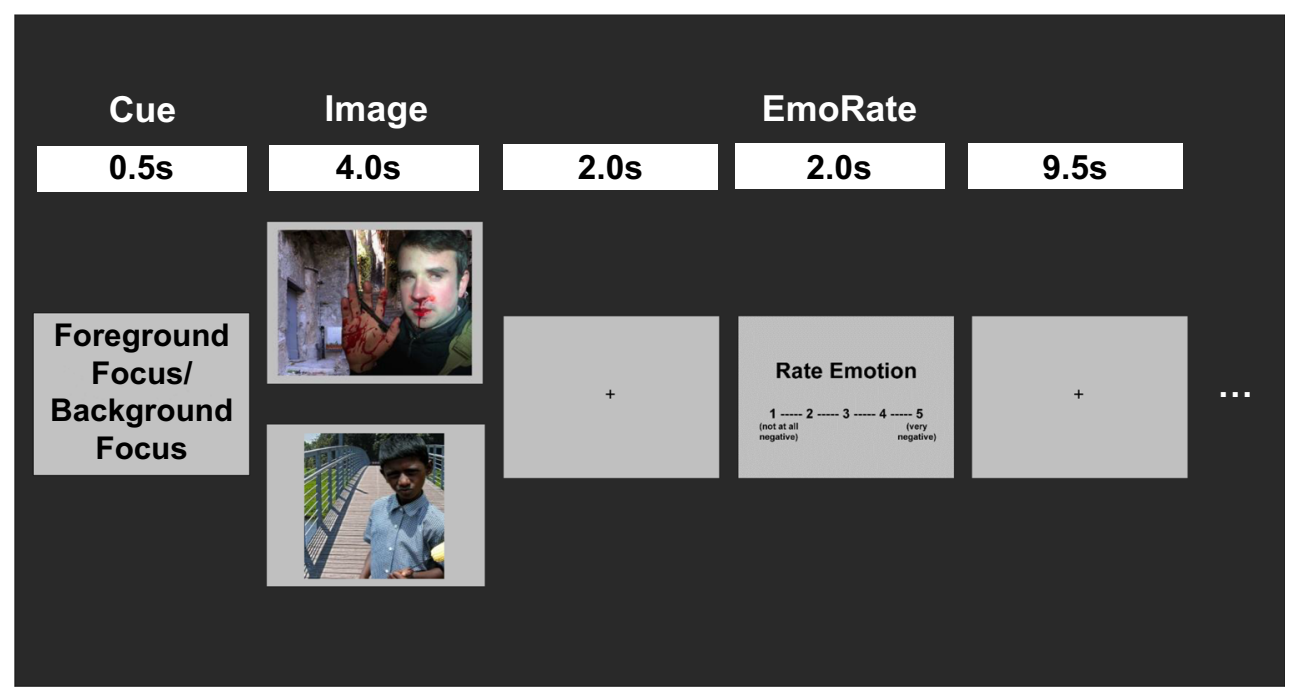

Figure 1. Diagram of the Emotional Rating Task with FA Manipulation. Participants were asked to view each image as cued by a preceding instruction screen (FG Focus or BG Focus). After viewing each image, participants rated their emotional experience on a 5-point scale (1 = "Not Negative at All"; 5 = "Very Negative"). 


\section{Manipulation Check and Behavioral Data Analyses}

To confirm that participants oriented their gaze as instructed, we calculated the proportion of time spent on fixations within the FG and $\mathrm{BG}$ areas for each trial using the EyeLink Data Viewer. These data were compared across Image Type (Negative vs. Neutral) and FA Manipulation (FG Focus vs. BG Focus) conditions. Similarly, to assess the behavioral impact of the FA manipulation on the subjective experience of emotion, repeated-measures analyses of variance (ANOVAs) and paired $t$-tests were performed to compare emotional ratings across Image Type (Negative vs. Neutral) and FA Manipulation (FG Focus vs. BG Focus) conditions. Behavioral and eye-tracking data were analyzed using the SPSS software (IBM Corp. 2017. Version 25.0); the effect sizes for the paired $t$-tests (Cohen's $d_{\mathrm{rm}}$ ) were calculated using the equations detailed by Lakens (2013).

\section{fMRI Data Analyses}

Preprocessing of the fMRI data was performed using SPM12 (Wellcome Department of Cognitive Neurology, London, UK). Functional images were first corrected for acquisition order and realigned to correct for motion artifacts. Next, the high-resolution anatomical image was co-registered with the first functional image for each participant, and functional images were spatially normalized (resampled to 2-mm isotropic voxels) to the Montreal Neurological Institute (MNI152) template. Lastly, the functional images were spatially smoothed using a 6-mm Gaussian kernel full-width-at-half-maximum (FWHM), to increase the signal-to-noise ratio. For subjectlevel analyses, evoked hemodynamic responses during the image presentation period in each trial were modeled by convolution with a canonical hemodynamic response function. These data were then analyzed in a general linear model framework using the attentional manipulation and the image type condition, as well as the six motion parameters as regressors.

For group-level analyses, targeted voxel-wise random-effects analyses were conducted to identify brain regions showing differential brain activation linked to the image type and attentional manipulation conditions. First, for replication purposes, given the present manipulation involving processing of emotional and neutral stimuli, brain regions involved in processing emotional visual stimuli were identified by contrasting the condition that produced the strongest emotional responses with the neutral condition (Emotional FG Focus > Neutral). Then, to investigate the effects of FA manipulation, and paralleling the behavioral comparisons, further targeted contrasts were performed as follows. The brain regions showing reduced response as a result of engaging FA as an ER strategy were identified by the contrasts between the
Emotional FG Focus minus Emotional BG Focus (EmoFG $>$ EmoBG). To make sure that this contrast identifies brain regions showing a reduction in activity linked to the FA manipulation in regions that are also engaged by emotion processing, the EmoFG $>$ EmoBG contrast was masked with the functional map identified by the contrast mentioned above for specifying brain regions involved in processing emotional visual stimuli $(\mathrm{EmoFG}>\mathrm{Neu})$. Finally, brain regions showing increased response as a result of engaging FA to focus away from the negative content of images were identified by the reverse contrast (EmoBG $>$ EmoFG). An intensity threshold of $p<0.005$ and an extent threshold of 46 contiguous voxels were used for the group-level targeted contrasts (e.g., EmoFG $>$ EmoBG, EmoBG $>$ EmoFG). These correspond to a threshold corrected for multiple comparisons at $p<0.05$, as determined by a Monte Carlo Simulation (1000 iterations; Slotnick, 2017; Slotnick, Moo, Segal, \& Hart, 2003). In the tables, we also denote results that survive a threshold clustercorrected for false discovery rate at $p<0.05$ (Benjamini \& Hochberg, 1995; Genovese, Lazar, \& Nichols, 2002). Additionally, an exploratory extent threshold of 30 contiguous voxels was employed, and notations were provided in the tables to indicate such exceptions (Allard \& Kensinger, 2014). Finally, in-house custom MATLAB scripts developed at Duke University and adapted for the purpose of the present investigation also were used to extract the time course of fMRI responses, as previously employed in published work (Denkova et al., 2010; Dolcos, Diaz-Granados, Wang, \& McCarthy, 2008; Dolcos et al., 2013; Dolcos \& McCarthy, 2006; Iordan \& Dolcos, 2017; Iordan et al., 2019; Morey et al., 2009).

\section{Results}

\section{Manipulation Check: Eye-tracking Results}

Supporting successful engagement of FA, eye-tracking data showed that participants directed their gaze toward the target components of both emotional and neutral images. Specifically, participants spent more time gazing within the FG areas when instructed to do so (FG Focus) than when instructed to focus on the BG areas (BG Focus), for both emotional $\left(t(20)=8.33, p<0.001, d_{r m}=2.60\right)$ and neutral $\left(t(20)=10.01, p<0.001, d_{r m}=3.51\right)$ images. Similarly, participants spent more time gazing within the $\mathrm{BG}$ areas when instructed to do so (BG Focus) than when instructed to focus on the FG areas (FG Focus), for both emotional $(t(20)=9.57$, $\left.p<0.001, d_{r m}=3.25\right)$ and neutral $(t(20)=10.10, p<0.001$, $\left.d_{r m}=3.74\right)$ images. Analyses of eye-tracking data also showed that participants were less able to focus on the neutral background when the corresponding foreground was negative. This effect was revealed by a repeated-measures 
ANOVA, with Image Type (negative, neutral) and Attentional Focus (FG focus, BG focus) as factors, showing a marginally significant interaction $\left(F(1,20)=4.29 ; p=0.052, \eta_{\mathrm{p}}{ }^{2}=0.18\right)$. Post hoc $t$-tests showed that gaze time within the FG area was significantly greater for negative than for neutral images under the BG focus $(t(20)=2.91, p=0.009)$, which may reflect increased difficulty to keep the attentional focus away from the emotional content of images (Dolcos et al., 2020a). Findings from fMRI data analyses provided further evidence that participants complied with task instructions (see below).

\section{Behavioral Results: Reduced Subjective Emotional Experience following Self-Guided FA}

Confirming our first prediction, there was an overall reduction in the emotional ratings for the BG Focus compared with FG Focus condition, and this was driven by reduced ratings for the negative images (Figure 2; Table 1). A repeated-measures ANOVA revealed a main effect of Image Type [Negative vs. Neutral; $\left.F(1,19)=577.98, p<0.001, \eta_{\mathrm{p}}{ }^{2}=0.97\right]$ and a main effect of Attentional Focus [FG Focus vs. BG Focus; $F(1,19)$ $\left.=212.51, p<0.001, \eta_{\mathrm{p}}{ }^{2}=0.92\right]$. There also was a significant Image Type $\times$ Attentional Focus interaction $[F(1,19)=$ $260.37, p<0.001, \eta_{\mathrm{p}}{ }^{2}=0.93$ ], which was driven by a significant reduction in ratings under BG Focus compared with FG Focus for negative images $\left[t(19)=-17.32, p<0.001, d_{r m}=\right.$ 4.66], but not for neutral images [ $t(19)=-0.04, p=0.97, d_{r m}=$ $-0.01]$.

\section{fMRI Results: Opposing Patterns of Response in Executive, Affective, and Perceptual Brain Regions, following FA}

First, for replication purposes, given the present manipulation involving processing of emotional and neutral stimuli, brain

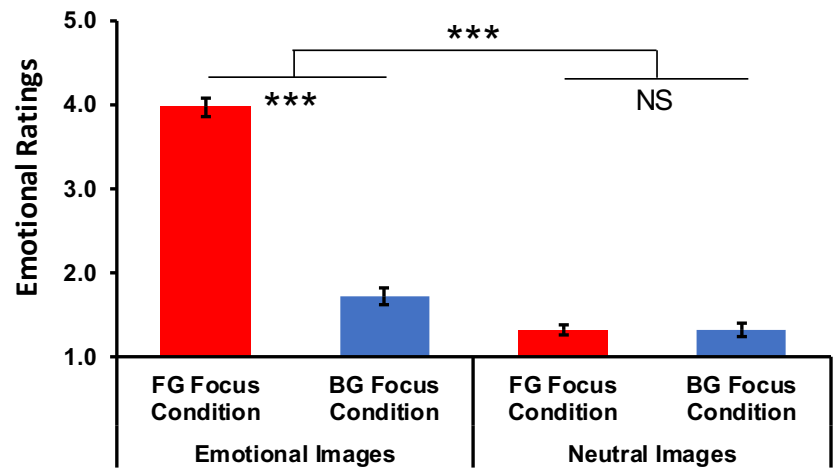

Figure 2. Reduced Emotional Ratings following FA Instructions. Instructions to focus on non-emotional contextual content (BG Focus) resulted in reduced emotional ratings, and this effect was driven by the emotional pictures. FG, Foreground; BG, Background; NS, not significant. Error bars indicate the standard error of the emotional rating means for each condition. $* * * p<0.001$.
Table 1. Eye-tracking and behavioral results

\begin{tabular}{llllll}
\hline & \multicolumn{3}{l}{ FG Focus } & & \multicolumn{2}{l}{ BG Focus } \\
\cline { 2 - 3 } \cline { 6 - 6 } \cline { 5 - 6 } & Negative & Neutral & & Negative & Neutral \\
\hline FG AI (\%) & $.71(.15)$ & $.71(.11)$ & $.34(.13)$ & $.31(.12)$ \\
BG AI (\%) & $.21(.09)$ & $.22(.07)$ & $.56(.13)$ & $.60(.13)$ \\
Emotional Ratings & $3.97(.50)$ & $1.32(.28)$ & $1.72(.46)$ & $1.32(.33)$ \\
\hline
\end{tabular}

Values indicate means (standard deviations). AI, area-of-interest; FG, foreground; BG, background.

regions involved in processing emotional visual stimuli were identified by contrasting the condition associated with increased emotion processing (Emotional FG Focus) and the neutral pictures condition $(\mathrm{EmoFG}>\mathrm{Neu})$. Consistent with previous studies, this analysis identified a host of brain regions that are part of the emotion processing network, such as the $\mathrm{AMY}$ and the ventrolateral PFC (vlPFC), as well as brain regions involved in visual processing that are also responsive to the emotional content of stimuli, such as the temporooccipital cortex and the fusiform gyrus (Table 2).

\section{Dissociable activity between executive vs. affective processing regions}

Confirming our second and third hypotheses, focusing on the contextual aspects of negative images was associated with increased activity in regions, typically implicated in topdown executive control, including the dIPFC and LPC, and with decreased activity in regions typically implicated in bottom-up emotion processing, including the AMY. Focusing on the contextual aspects was also associated with decreased activity in bilateral vlPFC (BA 45). Dissociable activity by FA also was identified in occipital regions, with superior areas showing increased activity and inferior occipital areas showing decreased activity when focusing on nonemotional contextual details of images (Figure 3; Tables 3-4).

\section{Dissociable activity in higher-order visual processing regions}

In addition to the dissociation between brain regions typically involved in executive vs. emotion processing, the engagement of self-guided FA was also linked to dissociable patterns of activity in regions involved in category-selective higher-order visual processing, with increased vs. decreased activity in the parahippocampal vs. fusiform gyri, respectively, when focusing on non-emotional contextual details of images (Figure 4). This provides further evidence that our task manipulation was indeed successful, as the BGs of images mainly consisted of scenes, and the FGs mainly consisted of faces. 
Table 2. Brain regions showing sensitivity to the emotional content of images

\begin{tabular}{|c|c|c|c|c|c|c|c|}
\hline \multirow[t]{2}{*}{ Brain region } & \multirow[t]{2}{*}{ Side } & \multirow[t]{2}{*}{ BA } & \multicolumn{3}{|c|}{ Talairach coordinates } & \multirow[t]{2}{*}{$\mathrm{t}$} & \multirow[t]{2}{*}{ Cluster size } \\
\hline & & & $\mathrm{x}$ & $\mathrm{y}$ & $\mathrm{z}$ & & \\
\hline \multicolumn{8}{|l|}{$E m o F G>\mathrm{Neu}$} \\
\hline \multicolumn{8}{|l|}{ Frontal/Insular Cortex } \\
\hline Middle frontal gyrus & $\mathrm{R}$ & 46 & 36 & 19 & 21 & 5.06 & 50 \\
\hline Inferior frontal gyrus & $\mathrm{L}$ & 44 & -42 & 0 & 23 & 4.42 & 97 \\
\hline Inferior frontal gyrus & $\mathrm{L}$ & 45 & -38 & 24 & 15 & 5.60 & $294 *$ \\
\hline Inferior frontal gyrus & $\mathrm{R}$ & 46 & 43 & 30 & 17 & 4.70 & $147^{*}$ \\
\hline Insula & $\mathrm{L}$ & 13 & -32 & 5 & -7 & 4.08 & $243 *$ \\
\hline Insula & $\mathrm{R}$ & 13 & 38 & 1 & -2 & 3.87 & $37 \dagger$ \\
\hline \multicolumn{8}{|l|}{ Parietal cortex } \\
\hline Postcentral gyrus & $\mathrm{L}$ & 40 & -53 & -34 & 33 & 5.08 & $256^{*}$ \\
\hline Postcentral gyrus & $\mathrm{R}$ & 40 & 57 & -27 & 30 & 5.24 & 70 \\
\hline Posterior cingulate & $\mathrm{L}$ & 23 & -9 & -50 & 21 & 3.97 & 56 \\
\hline \multicolumn{8}{|l|}{ Tempo-occipital cortex } \\
\hline Middle occipital gyrus & $\mathrm{L}$ & 39 & -53 & -62 & 8 & 7.20 & $850 *$ \\
\hline Inferior occipital gyrus & $\mathrm{L}$ & 19 & -38 & -72 & -12 & 3.77 & $107^{*}$ \\
\hline Superior occipital gyrus & $\mathrm{R}$ & 39 & 45 & -62 & 17 & 4.70 & $1521 *$ \\
\hline Middle occipital gyrus & $\mathrm{R}$ & 37 & 49 & -52 & 0 & 7.89 & \\
\hline Fusiform gyrus & $\mathrm{R}$ & 37 & 44 & -41 & -15 & 6.15 & \\
\hline Fusiform gyrus & $\mathrm{R}$ & 20 & 40 & -10 & -25 & 4.99 & 65 \\
\hline Fusiform gyrus & $\mathrm{L}$ & 37 & -39 & -39 & -16 & 4.80 & $209 *$ \\
\hline Parahippocampal gyrus & $\mathrm{L}$ & 36 & -28 & -6 & -25 & 5.28 & $44 \dagger$ \\
\hline \multicolumn{8}{|l|}{ Limbic and sublobar } \\
\hline Red nucleus & M & & 1 & -28 & -2 & 5.12 & $252 *$ \\
\hline Tectum & $\mathrm{R}$ & & 8 & -30 & -9 & 3.95 & \\
\hline Hypothalamus & $\mathrm{L}$ & & -6 & -6 & -9 & 4.89 & $147 *$ \\
\hline Amygdala & $\mathrm{L}$ & & -15 & -2 & -10 & 4.82 & \\
\hline Amygdala & $\mathrm{R}$ & & 15 & -4 & -8 & 4.14 & 60 \\
\hline Lateral globus pallidus & $\mathrm{R}$ & & 27 & -10 & -5 & 4.55 & \\
\hline Thalamus (VAN) & $\mathrm{L}$ & & -8 & -2 & 8 & 4.55 & $130 *$ \\
\hline
\end{tabular}

Peak coordinates for clusters were identified by random-effects analyses comparing the most emotional and neutral conditions (EmoFG $>$ Neu, clustercorrected for multiple comparisons; see Methods). EmoFG, Emotional-Foreground condition; Neu, Neutral condition; L, Left; R, Right; M, Medial. VAN, Ventral Anterior Nucleus. $†$ Significant at an exploratory extent threshold of 30 contiguous voxels. *Significant at the alternative cluster-extent threshold corrected for FDR at $p<0.05$ (see Methods).

\section{Discussion}

The present report investigated the neural mechanisms associated with the impact of self-guided FA on the subjective emotional experience triggered by viewing unpleasant pictures. There were three main novel findings. First, focusing on contextual aspects reduced subjective experience of emotions associated with the viewing of unpleasant pictures. Second, at the neural level, this behavioral effect was associated with opposing patterns of brain activity identifying increased vs. decreased responses in regions implicated in attentional/ executive control (e.g., dlPFC, LPC) vs. emotion processing (e.g., AMY, vlPFC). Third, dissociable patterns of brain activity were also identified in higher-order visual areas, showing increased vs. decreased responses in parahippocampal vs. fusiform gyri, respectively, when focusing on non-emotional contextual details of images. These findings are discussed in turn below.

\section{Reduced Subjective Emotional Experience following Self-Guided FA}

Extending available evidence regarding the effect of FA (Ferri et al., 2013; Urry, 2010), our results showed that focusing on the contextual aspects and away from the emotional aspects of negative (but not neutral) images decreased self-reported 


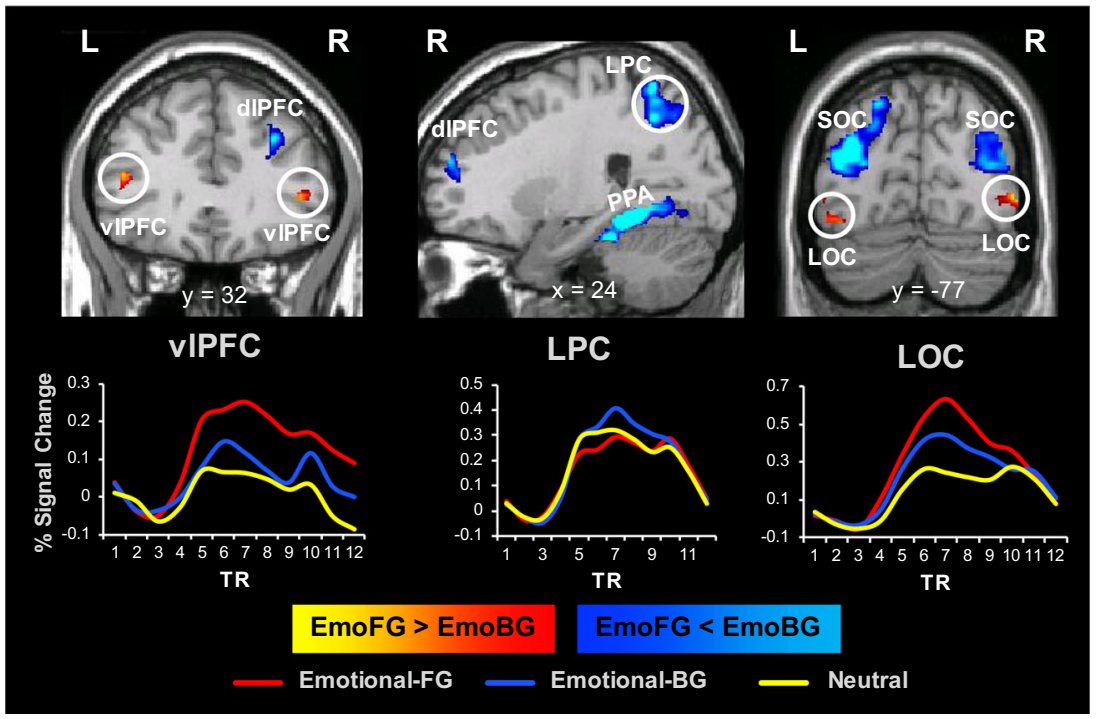

Figure 3. Dissociable activity in executive vs. emotion processing regions by the engagement of self-guided FA. Focusing on contextual aspects of negative images (BG Focus) was associated with increased activity in regions including the dIPFC, LPC, and superior occipital cortex (SOC), coupled with decreased activity in regions including the vlPFC, lateral occipital cortex (LOC), and amygdala (not shown). These activation maps were identified based on the Emotional-BG $>$ EmotionalFG and Emotional-FG > Emotional-BG contrasts, respectively (see Methods section); for illustration purposes, a voxel-wise intensity threshold of $p<0.01$ (uncorrected) was used. Time courses represent changes in the average fMRI signal extracted from peak and neighboring voxels; given the similarity in response in homologous regions across the two

emotional experiences associated with the viewing of such stimuli. This is overall consistent with recent theoretical frameworks that highlight the importance of the situational context in the effectiveness of ER strategies (Aldao, 2013; Aldao \& Tull, 2015). For example, in one study, participants' use of adaptive ER strategies was highly variable across different situations (e.g., social vs. achievement-related situations; Aldao \& Nolen-Hoeksema, 2012). Our results extend these models by demonstrating the efficacy of focusing on visual contextual details within a specific ER strategy, and its effect in reducing the associated negative emotional responses. These results complement evidence identified by our recent investigations (Denkova et al., 2015; Iordan et al., 2019) by showing that self-guided FA is effective not only in reducing negative emotional experiences associated with the recollection of unpleasant autobiographical memories, but also when employed while viewing unpleasant pictorial stimuli.

The effectiveness of attentional deployment strategies in regulating emotions has been demonstrated previously (Wadlinger \& Isaacowitz, 2011; Webb et al., 2012), most commonly in the form of cognitive distraction (e.g., diverting attention to something task-irrelevant; Oliver \& Page, 2008). However, some studies also identified the efficacy of externally guided FA in modulating emotional responses to pictorial stimuli (Ferri et al., 2013; Urry, 2010), but these latter hemispheres, the displayed fMRI signals were averaged from the left and right activation clusters. Notably, increased activity for the BG condition is equivalent to decreased activity for the FG condition. Hence, displaying the cold-colored clusters as reflecting the EmoFG $<$ EmoBG contrast allows consistency with the neuroimaging literature which typically shows increases using warm colors and decreases via cold colors. L, Left Hemisphere; R, Right Hemisphere; FG, Foreground; BG, Background; dlPFC, Dorsolateral Prefrontal Cortex; vlPFC, Ventrolateral Prefrontal Cortex; LPC, Lateral Parietal Cortex; SOC, Superior Occipital Cortex; LOC, Lateral Occipital Cortex; PPA, Parahippocampal Place Area.

studies employed experimental manipulations that involved the addition of guiding visual cues that do not exist in real life, thus reducing the ecological validity of the findings. By overcoming this limitation, and together with the findings from our studies of FA with internal emotional stimulation mentioned above, the present results show that self-guided FA can be a powerful tool in mitigating the subjective experience of negative emotions evoked by both internal and external emotional stimuli. This is important, because this ER strategy, which simply involved shifting of attentional focus within the same stimulus without forcibly directing participants where to look, does not require exhausting processing resources and thus can be easily deployed to cope with unexpected emotional challenges.

\section{Dissociable Activity between Executive vs. Affective Processing Regions, following FA}

Linked to these behavioral effects, the engagement of selfguided FA to focus on the contextual aspects of negative images was associated with increased activity in regions including the dIPFC, LPC, and SOC, coupled with decreased activity in regions including the AMY, vlPFC, and LOC. These findings are overall consistent with available evidence identifying the involvement of these regions linked to the 
Table 3. Brain regions showing increased activity when focusing on the contextual aspects of the negative images

\begin{tabular}{|c|c|c|c|c|c|c|c|}
\hline \multirow[t]{2}{*}{ Brain region } & \multirow[t]{2}{*}{ Side } & \multirow[t]{2}{*}{ BA } & \multicolumn{3}{|c|}{ Talairach coordinates } & \multirow[t]{2}{*}{$\mathrm{t}$} & \multirow[t]{2}{*}{ Cluster siz } \\
\hline & & & $\mathrm{x}$ & $\mathrm{y}$ & $\mathrm{z}$ & & \\
\hline \multicolumn{8}{|l|}{$\operatorname{EmoBG}>\operatorname{EmoFG}$} \\
\hline \multicolumn{8}{|l|}{ Frontal cortex } \\
\hline Frontal pole & $\mathrm{R}$ & 10 & 31 & 51 & -3 & 4.83 & $143 *$ \\
\hline Middle frontal gyrus & $\mathrm{R}$ & 11 & 25 & 44 & -7 & 4.42 & \\
\hline Superior frontal gyrus & $\mathrm{R}$ & 9 & 21 & 48 & 29 & 4.02 & $145 *$ \\
\hline Middle frontal gyrus & $\mathrm{R}$ & 10 & 30 & 45 & 22 & 4.34 & \\
\hline Superior frontal gyrus & $\mathrm{R}$ & 8 & 28 & 25 & 38 & 3.51 & 76 \\
\hline \multicolumn{8}{|l|}{ Parietal cortex } \\
\hline Superior parietal lobule & $\mathrm{L}$ & 7 & -24 & -59 & 47 & 4.89 & $148^{*}$ \\
\hline Supramarginal gyrus & $\mathrm{R}$ & 40 & 43 & -39 & 34 & 4.27 & 104 \\
\hline Precuneus & $\mathrm{R}$ & 7 & 18 & -61 & 55 & 5.04 & $512 *$ \\
\hline Precuneus & $\mathrm{L}$ & 7 & -6 & -59 & 54 & 2.87 & 77 \\
\hline Postcentral gyrus & $\mathrm{R}$ & 3 & 13 & -38 & 68 & 3.69 & $32 \dagger$ \\
\hline \multicolumn{8}{|l|}{ Temporo-occipital cortex } \\
\hline Middle temporal gyrus & $\mathrm{L}$ & 39 & -38 & -72 & 15 & 5.24 & $1079 *$ \\
\hline Superior occipital gyrus & $\mathrm{L}$ & 19 & -35 & -77 & 20 & 6.14 & \\
\hline Middle occipital gyrus & $\mathrm{R}$ & 19 & 32 & -78 & 17 & 4.27 & $377^{*}$ \\
\hline Precuneus & $\mathrm{R}$ & 31 & 30 & -70 & 25 & 3.84 & \\
\hline Cuneus & $\mathrm{R}$ & 18 & 13 & -86 & 23 & 5.26 & $411^{*}$ \\
\hline Cuneus & $\mathrm{R}$ & 17 & 6 & -87 & 9 & 4.64 & \\
\hline \multicolumn{8}{|l|}{ Limbic and subcortical } \\
\hline Posterior cingulate & $\mathrm{R}$ & 30 & 10 & -51 & 7 & 3.88 & $123 *$ \\
\hline Parahippocampal gyrus & $\mathrm{L}$ & 36 & -27 & -38 & -12 & 7.81 & $794 *$ \\
\hline Parahippocampal gyrus & $\mathrm{L}$ & 37 & -27 & -51 & -6 & 6.55 & \\
\hline Parahippocampal gyrus & $\mathrm{R}$ & 36 & 27 & -38 & -10 & 8.73 & $908 *$ \\
\hline Culmen & $\mathrm{R}$ & & 23 & -32 & -16 & 4.74 & \\
\hline
\end{tabular}

Peak coordinates for clusters were identified by the random-effects analyses comparing the BG and FG conditions for emotional images (EmoBG > EmoFG, cluster-corrected for multiple comparisons; see Methods). EmoBG, Emotional-Background condition; EmoFG, Emotional-Foreground condition. †Significant at an exploratory extent threshold of 30 contiguous voxels. *Significant at the alternative cluster-extent threshold corrected for FDR at $p<0.05$ (see Methods).

engagement of attentional deployment ER strategies, in general (Dorfel et al., 2014; Kanske, Heissler, Schonfelder, Bongers, \& Wessa, 2011; McRae et al., 2010), and in externally guided FA, in particular (Ferri et al., 2013). In addition, the observed opposing pattern of activity is also consistent with our prior work on emotional distraction, showing that reduced distraction is associated with incresed activity in executive regions and decreased activity in basic emotion processing regions; these regions are part of large-scale dorsal vs. ventral neural systems, respectively (Dolcos et al., 2006; Dolcos \& McCarthy, 2006; Dolcos et al., 2011; Iordan et al., 2013; Iordan et al., 2019). Notably, as previously emphasized (Iordan and Dolcos 2017; Iordan et al., 2019), there are considerable overlaps between these larger neural systems, identified through task manipulations of emotional distraction, and the large-scale functional networks, commonly identified through examination of resting-state functional connectivity-i.e., fronto-parietal/dorsal attention/centralexecutive vs. salience/ventral attention/cingulo-opercular, respectively (Corbetta and Shulman, 2002; Dosenbach et al., 2007; Dosenbach et al., 2006; Power et al., 2011; Seeley et al., 2007; Yeo et al., 2011). Hence, it is not surprising that voluntarily focusing attention away from emotionally salient information is associated with increased engagement of brain regions involved in top-down attentional control and reduced response in regions involved in bottom-up processing of saliency.

Due to the ability of emotional stimuli to rapidly "capture attention" and reallocate processing resources (Anderson \& Phelps, 2001; Pourtois, Schwartz, Seghier, Lazeyras, \& Vuilleumier, 2006; Shafer et al., 2012), it is possible that focusing away from the emotional aspects of negative images is effortful, resulting in greater engagement of brain regions associated with strategic top-down control. Voluntarily 
Table 4. Brain regions showing increased activation when focusing on the emotional aspects of the negative images

\begin{tabular}{|c|c|c|c|c|c|c|c|}
\hline \multirow[t]{2}{*}{ Brain region } & \multirow[t]{2}{*}{ Side } & \multirow[t]{2}{*}{ BA } & \multicolumn{3}{|c|}{ Talairach coordinates } & \multirow[t]{2}{*}{$\mathrm{t}$} & \multirow[t]{2}{*}{ Cluster size } \\
\hline & & & $\mathrm{x}$ & $\mathrm{y}$ & $\mathrm{z}$ & & \\
\hline \multicolumn{8}{|l|}{$E m o F G>E m o B G$} \\
\hline \multicolumn{8}{|l|}{ Frontal/insular cortex } \\
\hline Middle frontal gyrus & $\mathrm{L}$ & 46 & -42 & 25 & 20 & 4.20 & $101[261 *]$ \\
\hline Insula & $\mathrm{L}$ & 13 & -40 & 21 & 4 & 4.69 & \\
\hline Inferior frontal gyrus & $\mathrm{L}$ & 45 & -47 & 22 & 13 & 4.13 & \\
\hline Inferior frontal gyrus & $\mathrm{R}$ & 45 & 40 & 25 & 13 & 3.85 & $42 \dagger[44 \dagger]$ \\
\hline Anterior cingulate cortex & M & 24 & 5 & 25 & 0 & 3.81 & {$[75]$} \\
\hline Precentral gyrus & $\mathrm{L}$ & 44 & -49 & 11 & 10 & 3.95 & $82[261 *]$ \\
\hline \multicolumn{8}{|l|}{ Parietal cortex } \\
\hline Postcentral gyrus & $\mathrm{L}$ & 40 & -62 & -24 & 23 & 5.02 & $66[127]$ \\
\hline Postcentral gyrus & $\mathrm{L}$ & 2 & -59 & -24 & 37 & 4.74 & $69[88]$ \\
\hline \multicolumn{8}{|l|}{ Tempo-occipital cortex } \\
\hline Middle temporal gyrus & $\mathrm{L}$ & 37 & -53 & -60 & 7 & 4.40 & 92 \\
\hline Middle occipital gyrus & $\mathrm{L}$ & 19 & -7 & -54 & 22 & 4.34 & \\
\hline Middle occipital gyrus & $\mathrm{R}$ & 19 & 47 & -54 & 2 & 5.47 & $337 *[338 *]$ \\
\hline Inferior occipital gyrus & $\mathrm{L}$ & 18 & -36 & -81 & -13 & 4.19 & $86[163 *]$ \\
\hline Inferior occipital gyrus & $\mathrm{L}$ & 17 & -9 & -94 & -10 & 4.69 & {$[117]$} \\
\hline Inferior occipital gyrus & $\mathrm{R}$ & 17 & 21 & -94 & 1 & 3.71 & {$[82]$} \\
\hline Fusiform gyrus & $\mathrm{L}$ & 37 & -36 & -41 & -18 & 3.89 & $36 \dagger$ \\
\hline Fusiform gyrus & $\mathrm{R}$ & 37 & 43 & -42 & -13 & 5.34 & 100 \\
\hline \multicolumn{8}{|l|}{ Limbic and subcortical } \\
\hline Cingulate gyrus & $\mathrm{L}$ & 31 & -5 & -42 & 31 & 5.68 & $236 *[431 *]$ \\
\hline Posterior cingulate & M & 30 & 0 & -50 & 21 & 5.64 & \\
\hline Lentiform nucleus (Put.) & $\mathrm{L}$ & & -27 & 11 & -10 & 6.19 & $231 *[274 *]$ \\
\hline Claustrum & $\mathrm{L}$ & & -23 & 20 & -9 & 4.40 & \\
\hline Amygdala & $\mathrm{L}$ & & -16 & 1 & -9 & 4.23 & \\
\hline Thalamus (MDN) & $\mathrm{L}$ & & -7 & -17 & 8 & 4.09 & 97 [129] \\
\hline Thalamus (AN) & M & & -1 & 0 & 6 & 3.32 & \\
\hline \multicolumn{8}{|l|}{ Cerebellum } \\
\hline Tuber & $\mathrm{R}$ & & 25 & -80 & -28 & 4.82 & [89] \\
\hline Inferior semilunar lobule & $\mathrm{R}$ & & 27 & -77 & -35 & 3.76 & \\
\hline Uvula & $\mathrm{R}$ & & 36 & -74 & -26 & 3.13 & \\
\hline
\end{tabular}

Peak coordinates for clusters were identified by the random-effects analyses comparing the FG and BG focus conditions for emotional images (EmoFG $>$ EmoBG, cluster-corrected for multiple comparisons; see Methods), which was also inclusively masked with a statistical map showing increased activation for focusing on the emotional aspects of negative images relative to neutral images (EmoFG > Neu; see Methods); cluster sizes without the inclusion the EmoFG > Neu mask are noted in [square brackets]. EmoFG, Emotional-Foreground condition; EmoBG, Emotional-Background condition; Left; R, Right; M, Medial; Put., Putamen; MDN, Medial Dorsal Nucleus; AN, Anterior Nucleus. †Significant at an exploratory extent threshold of 30 contiguous voxels; *Significant at the alternative cluster-extent threshold corrected for FDR at $p<0.05$ (see Methods).

engaging FA requires increased recruitment of regions, such as the fronto-parietal regions, typically implicated in cognitive/executive control (Seeley et al., 2007; Vincent, Kahn, Snyder, Raichle, \& Buckner, 2008; Yeo et al., 2011). Although the current results do not allow for the interpretation of causality, it is possible that these regions that are important for executive control in turn modulate activity in emotion processing regions, such as the AMY and vlPFC, leading to the reduction in subjective emotional experience when viewing negative images by focusing on their context. Notably, previous studies have demonstrated functional heterogeneity in the vIPFC area (Cai et al., 2014; Gordon et al., 2016; Levy \& Wagner, 2011; Power et al., 2011; Warren et al., 2014), characterizing this region as a site of crossmodal integration involved in a wide range of affective and cognitive/executive processes. Available evidence points to the vlPFC's involvement not only in the generation and upregulation of emotion (Lindquist et al., 2012; Morawetz et al., 


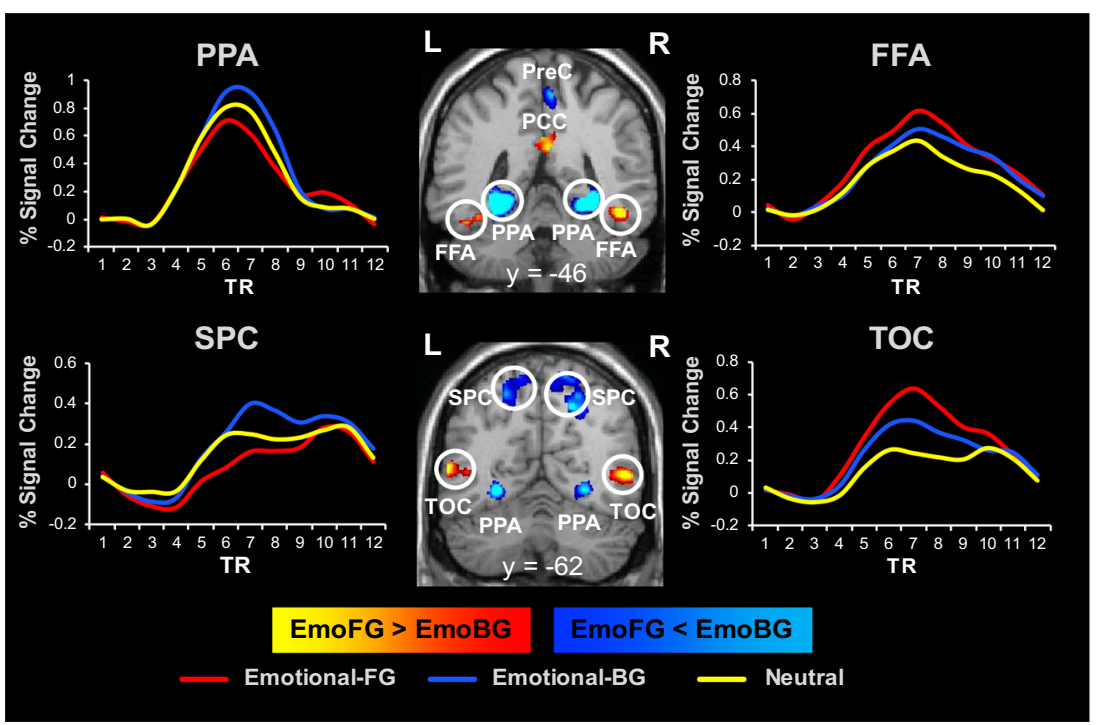

Figure 4. Dissociable activity in perceptual processing regions by the engagement of self-guided FA. Focusing on the contextual aspects of negative images was associated with increased vs. decreased activity in the parahippocampal place area (PPA) vs. fusiform face area (FFA) and temporo-occipital cortex (TOC), respectively. Additional dissociable activity was also observed in the medial posterior regions between the precuneus (PreC)/superior parietal cortex (SPC) and posterior cingulate cortex (PCC). These activation maps were identified based on the Emotional-BG $>$ Emotional-FG and Emotional-FG $>$ Emotional-BG contrasts, respectively (see Methods section); for illustration purposes, a voxel-wise intensity threshold of $p<0.01$ (uncorrected) was used. Time

2017; Phan et al., 2002; Wager et al., 2008) but also in cognitive control and emotion downregulation (Badre \& Wagner, 2007; Buhle et al., 2014; Iordan et al., 2013; Kober et al., 2008; Kohn et al., 2014; Ochsner et al., 2012). Therefore, another possibility is that increased vlPFC activity when focusing on the emotional aspects of the images may, in part, also reflect some form of less effortful ER, possibly to avoid negative imagery as a result of having to maintain attention on aversive material. This is consistent with available evidence showing that the vlPFC is involved in spontaneous coping with distracting emotions (Dolcos et al., 2006; Dolcos \& McCarthy, 2006).

Interestingly, together with evidence from other FA studies, the present findings also point to a possible dissociation in the PFC recruitment associated with the source of emotional stimuli. Specifically, a recent investigation from our group showed that focusing away from the emotional aspects during negative autobiographical memory recollection was associated with increased activity in the vmPFC, which mediated the link between AMY activity and emotional ratings of negative memories (Denkova et al., 2015). This finding, coupled with the present results involving the dIPFC, suggests the intriguing possibility that the engagement of self-guided FA to regulate emotional responses to internal (e.g., recollected personal memories) vs. external (e.g., viewed pictures) stimuli is subserved by partially dissociable mechanisms involving medial vs. lateral PFC regions, courses represent changes in the average fMRI signal extracted from peak and neighboring voxels; given the similarity in response in homologous regions across the two hemispheres, the displayed fMRI signals were averaged from the left and right activation clusters. Notably, increased activity for the BG condition is equivalent to decreased activity for the FG condition. Hence, displaying the cold-colored clusters as reflecting the EmoFG $<$ EmoBG contrast allows consistency with the neuroimaging literature which typically shows increases using warm colors and decreases via cold colors. L, Left Hemisphere; R, Right Hemisphere; FG, Foreground; BG, Background.

respectively. This is consistent with available evidence identifying associations between intrinsic functional connectivity of the medial vs. lateral anterior PFC and one's evaluations of their performance on memory vs. perceptual decision tasks, respectively (Baird, Smallwood, Gorgolewski, \& Margulies, 2013), thus pointing to differential contributions of the medial and lateral PFC subdivisions to internal and external modes of processing. Future studies of self-guided FA should clarify this issue by directly contrasting brain responses to internal and external stimuli within the same sample.

\section{Dissociable Activity in Higher-Order Visual Processing Regions, following FA}

In addition to the executive vs. affective dissociation discussed above, self-guided FA also was associated with opposing patterns of activity in higher-order visual processing regions (i.e., parahippocampal and fusiform gyri). On the one hand, focusing on the contextual aspects was associated with increased activity in the PPA, which is consistent with recent evidence regarding the involvement of this region in processing spatial and non-spatial contextual information (Diana, 2017) and with FA investigations from our group (Denkova et al., 2015; Iordan et al., 2019) identifying the involvement of this region in processing contextual aspects of recollected emotional autobiographical memories. On the other hand, focusing on the 
emotional aspects of negative images was associated with increased activity in the fusiform gyrus, which is consistent with available evidence identifying a specialized role of this region in face processing (Kanwisher, McDermott, \& Chun, 1997) as well as sensitivity to emotional facial expressions (Denkova et al., 2010; Kawasaki et al., 2012).

These findings raise an intriguing possibility that focusing on contextual vs. emotional aspects of negative pictorial material is linked to opposing modulations of category/domainspecific visual processing in these regions. However, because the FGs of the composite images often contained faces and the BGs represented scenes, the current experimental design does not allow us to determine the extent to which the observed dissociation in higher-order visual processing regions reflects top-down influences linked to the engagement of FA or differential specificity in basic perceptual processing of scenes vs. faces, respectively (Anderson, Christoff, Panitz, De Rosa, \& Gabrieli, 2003; Epstein \& Kanwisher, 1998). More research is needed to clarify this aspect, for instance, by examining how the degree of such dissociations in these higher-order perceptual regions relates to behavioral indices of selfguided FA. Nevertheless, this finding provides compelling evidence that participants did indeed comply with instructions to focus on the foreground or background aspects of images.

\section{Caveats}

One caveat of the present study is that the emotional content was always presented in the FG component of the negative images. To completely counterbalance the factors of interest, it would have been more ideal to also manipulate the emotional content of the BG areas. Although this may influence responses in specific brain regions (PPA vs. FFA), it does not limit the overall conclusion of the present study, given that we carefully selected and created our emotional and neutral composite stimuli, to allow a direct comparison of their effects. Other potential limitations are that the present study did not use positive emotional stimuli, and that the subject sample consisted solely of female participants, limiting the generalizability of the findings. Finally, given the relatively small sample of the current study, future studies of FA should aim for larger sample sizes, to reduce susceptibility to Type I and Type II errors (Cremers, Wager, \& Yarkoni, 2017). It is worth noting that sample sizes similar to that of the present studies are not uncommon in the broader ER literature (Kanske et al., 2011; McRae et al., 2010) and have yielded robust fMRI findings in previous FA studies (Denkova et al., 2015; Iordan et al., 2019).

\section{Conclusions}

The present study identified novel neurobehavioral effects of self-guided FA, shedding light on how simply focusing away from the emotional details of pictorial stimuli toward nonemotional contextual details modulates the subjective experience of emotions. At the behavioral level, self-guided FA reduced the subjective ratings of negative images. Paralleling this behavioral effect, fMRI results showed that focusing on the contextual aspects and away from the emotional aspects of negative images was associated with increased activity in executive regions as well as decreased activity in emotion processing regions. Engaging self-guided FA was also associated with modulations of activity in the parahippocampal and fusiform gyri, possibly reflecting domain-specific visuo-attentional and perceptual processing. Overall, our present results extend current evidence regarding the impact of attentional deployment strategies on subjective emotional experiences associated with processing of external stimuli, by showing that self-guided FA reduces negative emotional experience, possibly through increased involvement of top-down executive regions that modulate activity in bottom-up emotion processing regions. These findings advance our understanding of self-guided FA as an emotion control strategy and provide insights into possible ways of modulating the impact of distressing emotional stimuli to enhance emotional well-being (Dolcos et al., 2020c). Clarification of the neurobehavioral mechanisms of self-guided FA has important implications for understanding healthy functioning and affective disorders in which excessive attention to negative stimuli and rumination on negative memories often are among the core debilitating features.

Acknowledgments This work was conducted in part at the Biomedical Imaging Center of the Beckman Institute for Advanced Science and Technology at the University of Illinois at Urbana-Champaign (UIUCBI-BIC) and was supported by the UIUC Center for Social and Behavioral Science (to K.F.B., S.D., F.D., \& W.H). Participants were recruited from a larger study investigating mother-child dyads during early child development, which was supported in part by grants from the National Dairy Council (to Sharon Donovan and Barbara H. Fiese (Co-PIs), the Gerber Foundation (to Sharon Donovan), the Christopher Family Foundation (to Sharon Donovan and K.F.B), the US Department of Agriculture (Hatch ILLU 793-330, to Barbara H. Fiese, K.F.B., and Margarita Teran-Garcia), and the National Institutes of Health (DK107561, to Sharon Donovan). The authors wish to thank JulieAnn Scherer, Michael Budianto, and other Dolcos Lab members for help with the creation of stimuli, and Samantha Iwinski, Jaclyn Theisen, and Jaclyn Saltzman for help with participant recruitment.

Author Contributions F.D. \& S.D. designed the study; Y.K. and R.L implemented the MRI/Eye-tracking recordings, with input from F.D. and S.D.; Y.K. and C.S. collected the data; F.D., S.D., and Y.K. planned the analytical approach; C.S., Y.K., S.J., and P.C.B. performed the analyses, with input from F.D. and S.D.; F.D., S.D., Y.K., C.S., S.J., and P.C.B. contributed to the writing of the manuscript, with feedback from W.H. and K.F.B.

\section{References}

Aldao, A. (2013). The Future of Emotion Regulation Research: Capturing Context. Perspect Psychol Sci, 8(2), 155-172. https:// doi.org/10.1177/1745691612459518 
Aldao, A., \& Nolen-Hoeksema, S. (2012). The influence of context on the implementation of adaptive emotion regulation strategies. Behav Res Ther, 50(7-8), 493-501. https://doi.org/10.1016/j.brat. 2012.04.004

Aldao, A., \& Tull, M. T. (2015). Putting emotion regulation in context. Current Opinion in Psychology, 3, 100-107. https://doi.org/10. 1016/j.copsyc. 2015.03 .022

Allard, E. S., \& Kensinger, E. A. (2014). Age-related differences in neural recruitment during the use of cognitive reappraisal and selective attention as emotion regulation strategies. Front Psychol, 5, 296. https://doi.org/10.3389/fpsyg.2014.00296

Anderson, A. K., Christoff, K., Panitz, D., De Rosa, E., \& Gabrieli, J. D. (2003). Neural correlates of the automatic processing of threat facial signals. J Neurosci, 23(13), 5627-5633. Retrieved from http://www. ncbi.nlm.nih.gov/pubmed/12843265

Anderson, A. K., \& Phelps, E. A. (2001). Lesions of the human amygdala impair enhanced perception of emotionally salient events. Nature, 411(6835), 305-309. https://doi.org/10.1038/35077083

Anticevic, A., Repovs, G., \& Barch, D. M. (2010). Resisting emotional interference: brain regions facilitating working memory performance during negative distraction. Cognitive, affective \& behavioral neuroscience, 10(2), 159-173. https://doi.org/10.3758/ CABN.10.2.159

Badre, D., \& Wagner, A. D. (2007). Left ventrolateral prefrontal cortex and the cognitive control of memory. Neuropsychologia, 45(13), 2883-2901. https://doi.org/10.1016/j.neuropsychologia.2007.06. 015

Baird, B., Smallwood, J., Gorgolewski, K. J., \& Margulies, D. S. (2013). Medial and Lateral Networks in Anterior Prefrontal Cortex Support Metacognitive Ability for Memory and Perception. The Journal of Neuroscience, 33(42), 16657-16665. https://doi.org/10.1523/ jneurosci.0786-13.2013

Benjamini, Y., \& Hochberg, Y. (1995). Controlling the false discovery rate: a practical and powerful approach to multiple testing. Journal of the Royal statistical society: series B (Methodological), 57(1), 289-300.

Buhle, J. T., Silvers, J. A., Wager, T. D., Lopez, R., Onyemekwu, C., Kober, H., ... Ochsner, K. N. (2014). Cognitive reappraisal of emotion: a meta-analysis of human neuroimaging studies. Cerebral cortex, 24(11), 2981-2990.

Cai, W., Ryali, S., Chen, T., Li, C. S., \& Menon, V. (2014). Dissociable roles of right inferior frontal cortex and anterior insula in inhibitory control: evidence from intrinsic and task-related functional parcellation, connectivity, and response profile analyses across multiple datasets. J Neurosci, 34(44), 14652-14667. https://doi.org/10. 1523/JNEUROSCI.3048-14.2014

Corbetta M, Shulman GL. 2002. Control of goal-directed and stimulusdriven attention in the brain. Nat Rev Neurosci. 3 (3):201-215.

Cremers, H. R., Wager, T. D., \& Yarkoni, T. (2017). The relation between statistical power and inference in fMRI. PLoS One, 12(11), e0184923. https://doi.org/10.1371/journal.pone.0184923

Dan-Glauser, E. S., \& Scherer, K. R. (2011). The Geneva affective picture database (GAPED): a new 730-picture database focusing on valence and normative significance. Behavior Research Methods, 43(2), 468-477. https://doi.org/10.3758/s13428-011-0064-1

Denkova, E., Dolcos, S., \& Dolcos, F. (2013a). The Effect of Retrieval Focus and Emotional Valence on the Inferior Frontal Cortex Activity during Autobiographical Recollection. Front Behav Neurosci, 7, 192. https://doi.org/10.3389/fnbeh.2013.00192

Denkova, E., Dolcos, S., \& Dolcos, F. (2013b). The Effect of Retrieval Focus and Emotional Valence on the Medial Temporal Lobe Activity during Autobiographical Recollection. Front Behav Neurosci, 7, 109. https://doi.org/10.3389/fnbeh.2013.00109

Denkova, E., Dolcos, S., \& Dolcos, F. (2015). Neural correlates of 'distracting' from emotion during autobiographical recollection.
Soc Cogn Affect Neurosci, 10(2), 219-230. https://doi.org/10.1093/ scan/nsu039

Denkova, E., Wong, G., Dolcos, S., Sung, K., Wang, L., Coupland, N., \& Dolcos, F. (2010). The impact of anxiety-inducing distraction on cognitive performance: a combined brain imaging and personality investigation. PLoS One, 5(11), e14150. https://doi.org/10.1371/ journal.pone.0014150

Diana, R. A. (2017). Parahippocampal Cortex Processes the Nonspatial Context of an Event. Cereb Cortex, 27(3), 1808-1816. https://doi. org/10.1093/cercor/bhw014

Dolcos, F., Bogdan, P., O'Brien, M., Iordan, A. D., Madison, A., Buetti, S., ... Dolcos, S. (2020a, in press). The Impact of Focused Attention on Emotional Evaluation: An Eye-Tracking Investigation. Emotion, in press.

Dolcos, F., Diaz-Granados, P., Wang, L., \& McCarthy, G. (2008). Opposing influences of emotional and non-emotional distracters upon sustained prefrontal cortex activity during a delayed-response working memory task. Neuropsychologia, 46(1), 326-335. https:// doi.org/10.1016/j.neuropsychologia.2007.07.010

Dolcos, F., Iordan, A. D., \& Dolcos, S. (2011). Neural correlates of emotion-cognition interactions: A review of evidence from brain imaging investigations. J Cogn Psychol (Hove), 23(6), 669-694. https://doi.org/10.1080/20445911.2011.594433

Dolcos, F., Iordan, A. D., Kragel, J., Stokes, J., Campbell, R., McCarthy, G., \& Cabeza, R. (2013). Neural correlates of opposing effects of emotional distraction on working memory and episodic memory: an event-related FMRI investigation. Front Psychol, 4, 293. https://doi. org/10.3389/fpsyg.2013.00293

Dolcos, F., Katsumi, Y., Bogdan, P., Shen, C., Jun, S., Buetti, S., ... Dolcos, S. (2020b). The impact of Focused Attention on subsequent emotional recollection: A functional MRI investigation. Neuropsychologia, 107338.

Dolcos, F., Katsumi, Y., Moore, M., Berggren, N., de Gelder, B., Derakshan, N., ... \& Dolcos, S. (2020c). Neural correlates of emotion-attention interactions: From perception, learning, and memory to social cognition, individual differences, and training interventions. Neuroscience \& Biobehavioral Reviews, 108, 559-601.

Dolcos, F., Katsumi, Y., Weymar, M., Moore, M., Tsukiura, T., \& Dolcos, S. (2017). Emerging directions in emotional episodic memory. Frontiers in Psychology, 8, 1867. https://doi.org/10.3389/ fpsyg.2017.01867

Dolcos, F., Kragel, P., Wang, L., \& McCarthy, G. (2006). Role of the inferior frontal cortex in coping with distracting emotions. Neuroreport, 17(15), 1591-1594. https://doi.org/10.1097/01.wnr. 0000236860.24081.be

Dolcos, F., \& McCarthy, G. (2006). Brain systems mediating cognitive interference by emotional distraction. J Neurosci, 26(7), 2072-2079. https://doi.org/10.1523/JNEUROSCI.5042-05.2006

Dorfel, D., Lamke, J. P., Hummel, F., Wagner, U., Erk, S., \& Walter, H. (2014). Common and differential neural networks of emotion regulation by Detachment, Reinterpretation, Distraction, and Expressive Suppression: a comparative fMRI investigation. Neuroimage, 101, 298-309. https://doi.org/10.1016/j.neuroimage.2014.06.051

Dosenbach, N. U., Fair, D. A., Miezin, F. M., Cohen, A. L., Wenger, K. K., Dosenbach, R. A., ... Petersen, S. E. (2007). Distinct brain networks for adaptive and stable task control in humans. Proc Natl Acad Sci U S A, 104(26), 11073-11078. https://doi.org/10.1073/ pnas.0704320104

Dosenbach, N. U., Visscher, K. M., Palmer, E. D., Miezin, F. M., Wenger, K. K., Kang, H. C., ... Petersen, S. E. (2006). A core system for the implementation of task sets. Neuron, 50(5), 799812. https://doi.org/10.1016/j.neuron.2006.04.031

Epstein, R., \& Kanwisher, N. (1998). A cortical representation of the local visual environment. Nature, 392(6676), 598-601. https://doi. org $/ 10.1038 / 33402$ 
Ferri, J., Schmidt, J., Hajcak, G., \& Canli, T. (2013). Neural correlates of attentional deployment within unpleasant pictures. Neuroimage, 70 , 268-277. https://doi.org/10.1016/j.neuroimage.2012.12.030

Genovese, C. R., Lazar, N. A., \& Nichols, T. (2002). Thresholding of statistical maps in functional neuroimaging using the false discovery rate. Neuroimage, 15(4), 870-878.

Goodman, A. M., Katz, J. S., \& Dretsch, M. N. (2016). Military Affective Picture System (MAPS): A new emotion-based stimuli set for assessing emotional processing in military populations. Journal of Behavior Therapy and Experimental Psychiatry, 50, 152-161. https://doi.org/10.1016/j.jbtep.2015.07.006

Gordon, E. M., Laumann, T. O., Adeyemo, B., Huckins, J. F., Kelley, W. M., \& Petersen, S. E. (2016). Generation and Evaluation of a Cortical Area Parcellation from Resting-State Correlations. Cereb Cortex, 26(1), 288-303. https://doi.org/10.1093/cercor/bhu239

Gross, J. J. (1998). The Emerging Field of Emotion Regulation: An Integrative Review. Review of General Psychology, 2(3), 271-299. https://doi.org/10.1037/1089-2680.2.3.271

Gross, J. J. (2008). Emotion regulation. In M. Lewis, J. M. HavilandJones, \& L. F. Barrett (Eds.), Handbook of emotions (pp. 497-512). New York: Guilford.

Gross, J. J. (2015). Emotion regulation: Current status and future prospects. Psychological Inquiry, 26(1), 1-26. https://doi.org/10.1080/ 1047840x.2014.940781

Iordan, A. D., \& Dolcos, F. (2017). Brain Activity and Network Interactions Linked to Valence-Related Differences in the Impact of Emotional Distraction. Cereb Cortex, 27(1), 731-749. https:// doi.org/10.1093/cercor/bhv242

Iordan, A. D., Dolcos, S., \& Dolcos, F. (2013). Neural signatures of the response to emotional distraction: a review of evidence from brain imaging investigations. Front Hum Neurosci, 7, 200. https://doi.org/ 10.3389 /fnhum. 2013.00200

Iordan, A. D., Dolcos, S., \& Dolcos, F. (2019). Brain Activity and Network Interactions in the Impact of Internal Emotional Distraction. Cereb Cortex, 29(6), 2607-2623. https://doi.org/10. 1093/cercor/bhy129

Johnstone, K. A., \& Page, A. C. (2004). Attention to phobic stimuli during exposure: The effect of distraction on anxiety reduction, self-efficacy and perceived control. Behaviour research and therapy, 42(3), 249-275.

Kanske, P., Heissler, J., Schonfelder, S., Bongers, A., \& Wessa, M. (2011). How to regulate emotion? Neural networks for reappraisal and distraction. Cereb Cortex, 21(6), 1379-1388. https://doi.org/10. 1093/cercor/bhq216

Kanwisher, N., McDermott, J., \& Chun, M. M. (1997). The fusiform face area: a module in human extrastriate cortex specialized for face perception. J Neurosci, 17(11), 4302-4311. Retrieved from http:// www.ncbi.nlm.nih.gov/pubmed/9151747

Kawasaki, H., Tsuchiya, N., Kovach, C. K., Nourski, K. V., Oya, H., Howard, M. A., \& Adolphs, R. (2012). Processing of facial emotion in the human fusiform gyrus. J Cogn Neurosci, 24(6), 1358-1370. https://doi.org/10.1162/jocn a 00175

Kober, H., Barrett, L. F., Joseph, J., Bliss-Moreau, E., Lindquist, K., \& Wager, T. D. (2008). Functional grouping and cortical-subcortical interactions in emotion: a meta-analysis of neuroimaging studies. Neuroimage, 42(2), 998-1031.

Kohn, N., Eickhoff, S. B., Scheller, M., Laird, A. R., Fox, P. T., \& Habel, U. (2014). Neural network of cognitive emotion regulation-an ALE meta-analysis and MACM analysis. Neuroimage, 87, 345-355. https://doi.org/10.1016/j.neuroimage.2013.11.001

Lakens, D. (2013). Calculating and reporting effect sizes to facilitate cumulative science: a practical primer for t-tests and ANOVAs. Front Psychol, 4, 863. https://doi.org/10.3389/fpsyg.2013.00863

Lang, P. J., Bradley, M. M., \& Cuthbert, B. N. (2008). International affective picture system (IAPS): Affective ratings of pictures and instruction manual. Retrieved from
Levy, B. J., \& Wagner, A. D. (2011). Cognitive control and right ventrolateral prefrontal cortex: reflexive reorienting, motor inhibition, and action updating. Ann N Y Acad Sci, 1224, 40-62. https://doi.org/10. 1111/j.1749-6632.2011.05958.x

Lindquist, K. A., Wager, T. D., Kober, H., Bliss-Moreau, E., \& Barrett, L. F. (2012). The brain basis of emotion: A meta-analytic review. Behavioral and Brain Sciences, 35(3), 121-143. https://doi.org/10. 1017/S0140525X11000446

Marchewka, A., Zurawski, L., Jednorog, K., \& Grabowska, A. (2014). The Nencki Affective Picture System (NAPS): Introduction to a novel, standardized, wide-range, high-quality, realistic picture database. Behavior Research Methods, 46(2), 596-610. https://doi.org/ 10.3758/s13428-013-0379-1

McRae, K., Hughes, B., Chopra, S., Gabrieli, J. D., Gross, J. J., \& Ochsner, K. N. (2010). The neural bases of distraction and reappraisal. J Cogn Neurosci, 22(2), 248-262. https://doi.org/10.1162/ jocn.2009.21243

Morawetz, C., Bode, S., Derntl, B., \& Heekeren, H. R. (2017). The effect of strategies, goals and stimulus material on the neural mechanisms of emotion regulation: A meta-analysis of fMRI studies. Neurosci Biobehav Rev, 72, 111-128. https://doi.org/10.1016/j.neubiorev. 2016.11.014

Morey, R. A., Dolcos, F., Petty, C. M., Cooper, D. A., Hayes, J. P., LaBar, K. S., \& McCarthy, G. (2009). The role of trauma-related distractors on neural systems for working memory and emotion processing in posttraumatic stress disorder. J Psychiatr Res, 43(8), 809-817. https://doi.org/10.1016/j.jpsychires.2008.10.014

Nolen-Hoeksema, S., Wisco, B. E., \& Lyubomirsky, S. (2008). Rethinking Rumination. Perspect Psychol Sci, 3(5), 400-424. https://doi.org/10.1111/j.1745-6924.2008.00088.x

Ochsner, K. N., Silvers, J. A., \& Buhle, J. T. (2012). Functional imaging studies of emotion regulation: a synthetic review and evolving model of the cognitive control of emotion. Annals of the New York Academy of Sciences, 1251, E1-24. https://doi.org/10.1111/j.17496632.2012.06751.x

Oliver, N. S., \& Page, A. C. (2003). Fear reduction during in vivo exposure to blood-injection stimuli: Distraction vs. attentional focus. British Journal of Clinical Psychology, 42(1), 13-25.

Oliver, N. S., \& Page, A. C. (2008). Effects of internal and external distraction and focus during exposure to blood-injury-injection stimuli. Journal of Anxiety Disorders, 22(2), 283-291.

Paul, S., Simon, D., Kniesche, R., Kathmann, N., \& Endrass, T. (2013). Timing effects of antecedent- and response-focused emotion regulation strategies. Biological Psychology, 94(1), 136-142. https://doi. org/10.1016/j.biopsycho.2013.05.019

Phan, K. L., Fitzgerald, D. A., Nathan, P. J., Moore, G. J., Uhde, T. W., \& Tancer, M. E. (2005). Neural substrates for voluntary suppression of negative affect: a functional magnetic resonance imaging study. Biol Psychiatry, 57(3), 210-219. https://doi.org/10.1016/j.biopsych. 2004.10.030

Phan, K. L., Wager, T., Taylor, S. F., \& Liberzon, I. (2002). Functional Neuroanatomy of Emotion: A Meta-Analysis of Emotion Activation Studies in PET and fMRI. Neuroimage, 16(2), 331-348. https://doi. org/10.1006/nimg.2002.1087

Pourtois, G., Schwartz, S., Seghier, M. L., Lazeyras, F., \& Vuilleumier, P. (2006). Neural systems for orienting attention to the location of threat signals: an event-related fMRI study. Neuroimage, 31(2), 920-933. https://doi.org/10.1016/j.neuroimage.2005.12.034

Power, J. D., Cohen, A. L., Nelson, S. M., Wig, G. S., Barnes, K. A., Church, J. A., ... Petersen, S. E. (2011). Functional network organization of the human brain. Neuron, 72(4), 665-678. https://doi.org/ 10.1016/j.neuron.2011.09.006

Scheibe, S., Sheppes, G., \& Staudinger, U. M. (2015). Distract or reappraise? Age-related differences in emotion-regulation choice. Emotion, 15(6), 677-681. https://doi.org/10.1037/a0039246 
Seeley, W. W., Menon, V., Schatzberg, A. F., Keller, J., Glover, G. H., Kenna, H., ... Greicius, M. D. (2007). Dissociable intrinsic connectivity networks for salience processing and executive control. $J$ Neurosci, 27(9), 2349-2356. https://doi.org/10.1523/jneurosci. 5587-06.2007

Seo, S. (2006). A review and comparison of methods for detecting outliers in univariate data sets. University of Pittsburgh,

Shafer, A. T., Matveychuk, D., Penney, T., O'Hare, A. J., Stokes, J., \& Dolcos, F. (2012). Processing of emotional distraction is both automatic and modulated by attention: evidence from an event-related fMRI investigation. J Cogn Neurosci, 24(5), 1233-1252. https://doi. org/10.1162/jocn_a_00206

Sheppes, G., \& Meiran, N. (2007). Better late than never? On the dynamics of online regulation of sadness using distraction and cognitive reappraisal. Personality \& Social Psychology Bulletin, 33(11), 1518-1532. https://doi.org/10.1177/0146167207305537

Sheppes, G., Scheibe, S., Suri, G., \& Gross, J. J. (2011). Emotionregulation choice. Psychol Sci, 22(11), 1391-1396. https://doi.org/ 10.1177/0956797611418350

Sheppes, G., Scheibe, S., Suri, G., Radu, P., Blechert, J., \& Gross, J. J. (2014). Emotion regulation choice: a conceptual framework and supporting evidence. Journal of Experimental Psychology. General, 143(1), 163-181. https://doi.org/10.1037/a0030831

Sheppes, G., Suri, G., \& Gross, J. J. (2015). Emotion Regulation and Psychopathology. Annual Review of Clinical Psychology, 11(1), 379-405. https://doi.org/10.1146/annurev-clinpsy-032814-112739

Slotnick, S. D. (2017). Resting-state fMRI data reflects default network activity rather than null data: A defense of commonly employed methods to correct for multiple comparisons. Cognitive Neuroscience, 8(3), 141-143. https://doi.org/10.1080/17588928. 2016.1273892

Slotnick, S. D., Moo, L. R., Segal, J. B., \& Hart, J. (2003). Distinct prefrontal cortex activity associated with item memory and source memory for visual shapes. Cognitive Brain Research, 17(1), 75-82. https://doi.org/10.1016/S0926-6410(03)00082-X

Thiruchselvam, R., Blechert, J., Sheppes, G., Rydstrom, A., \& Gross, J. J. (2011). The temporal dynamics of emotion regulation: An EEG study of distraction and reappraisal. Biological Psychology, 87(1), 84-92. https://doi.org/10.1016/j.biopsycho.2011.02.009

Urry, H. L. (2009). Using reappraisal to regulate unpleasant emotional episodes: goals and timing matter. Emotion, 9(6), 782-797. https:// doi.org/10.1037/a0017109
Urry, H. L. (2010). Seeing, thinking, and feeling: emotion-regulating effects of gaze-directed cognitive reappraisal. Emotion, 10(1), 125135. https://doi.org/10.1037/a0017434

Vincent, J. L., Kahn, I., Snyder, A. Z., Raichle, M. E., \& Buckner, R. L. (2008). Evidence for a frontoparietal control system revealed by intrinsic functional connectivity. Journal of neurophysiology, 100(6), 3328-3342. https://doi.org/10.1152/jn.90355.2008

Wadlinger, H. A., \& Isaacowitz, D. M. (2011). Fixing our focus: training attention to regulate emotion. Pers Soc Psychol Rev, 15(1), 75-102. https://doi.org/10.1177/1088868310365565

Wager, T. D., Davidson, M. L., Hughes, B. L., Lindquist, M. A., \& Ochsner, K. N. (2008). Prefrontal-subcortical pathways mediating successful emotion regulation. Neuron, 59(6), 1037-1050. https:// doi.org/10.1016/j.neuron.2008.09.006

Warren, D. E., Power, J. D., Bruss, J., Denburg, N. L., Waldron, E. J., Sun, H., ... Tranel, D. (2014). Network measures predict neuropsychological outcome after brain injury. Proc Natl Acad Sci U S A, 111(39), 14247-14252. https://doi.org/10.1073/pnas.1322173111

Webb, T. L., Miles, E., \& Sheeran, P. (2012). Dealing with feeling: a meta-analysis of the effectiveness of strategies derived from the process model of emotion regulation. Psychol Bull, 138(4), 775808. https://doi.org/10.1037/a0027600

Wessa, M., Kanske, P., Neumeister, P., Bode, K., Heissler, J., \& Schönfelder, S. (2010). EmoPicS: subjective and psychophysiological evaluation of new imagery for clinical biopsychological research. Z. Klin. Psychol. Psychother. Suppl, 1, 11-77.

Yeo, B. T. T., Krienen, F. M., Sepulcre, J., Sabuncu, M. R., Lashkari, D., Hollinshead, M., ... Buckner, R. L. (2011). The organization of the human cerebral cortex estimated by intrinsic functional connectivity. Journal of neurophysiology, 106(3), 1125-1165. https://doi.org/ 10.1152/jn.00338.2011.

Open Practices Statement The data and materials for this study will be made available upon request. This study was not preregistered.

Publisher's note Springer Nature remains neutral with regard to jurisdictional claims in published maps and institutional affiliations. 